\title{
Fbxw7 regulates renal cell carcinoma migration and invasion via suppression of the epithelial-mesenchymal transition
}

\author{
HONGCHAO HE ${ }^{*}$, JUN DAI* ${ }^{*}$ ZHAOPING XU, WEI HE, XIAOJING WANG, YU ZHU and HAOFEI WANG \\ Department of Urology, Ruijin Hospital, Shanghai Jiao Tong University School of Medicine, Shanghai 200025, P.R. China
}

Received August 18, 2016; Accepted November 7, 2017

DOI: $10.3892 / \mathrm{ol} .2018 .7744$

\begin{abstract}
F-box and WD repeat domain containing 7 (Fbxw7) is an F-box protein that belongs to the SKP1-CUL1-F-box protein E3 ligase complex and is responsible for transferring the ubiquitin molecule to the substrate, which results in its recognition and subsequent degradation by proteasomes. Furthermore, it can identify a network of signaling proteins that function in cell growth, diversion and apoptosis. In the present study, Fbxw7 was downregulated in renal cell carcinoma (RCC) tissues compared with the adjacent non-tumor tissues and its expression was significantly associated with the tumor-node-metastasis stage, lymph node metastasis and distant metastasis in patients with RCC. Furthermore, multivariate Cox regression analyses indicated that Fbxw7 expression was an independent factor for the prediction of the overall survival of patients with RCC. A functional study demonstrated that downregulation of Fbxw7 facilitated tumor cell migration and invasion via the epithelial-mesenchymal transition (EMT). Therefore, the results of the current study indicted that Fbxw7 is an anti-oncogene that serves a notable function in RCC development by suppressing RCC metastasis and the EMT, indicating the potential therapeutic value of Fbxw7 in inhibiting metastasis in RCC.
\end{abstract}

\section{Introduction}

Renal cell carcinoma (RCC) accounts for 2-3\% of all malignancies in adults and is the most common type of tumor of the urinary system. RCCs may be divided into clear cell,

Correspondence to: Dr Haofei Wang, Department of Urology, Ruijin Hospital, Shanghai Jiao Tong University School of Medicine, 197 Ruijin 2nd Road, Shanghai 200025, P.R. China

E-mail:wanglog@sina.com

*Contributed equally

Abbreviations: RCC, renal cell carcinoma; EMT, epithelial-mesenchymal transition; Fbxw7, F-box and WD repeat domain containing 7; IHC, immunohistochemistry; siRNA, small interfering RNA; OS, overall survival

Key words: renal cell carcinoma, Fbxw7, epithelial-mesenchymal transition, metastasis, invasion papillary, chromophobe, collecting duct and unclassified RCC (1). Renal clear cell carcinoma, originating from renal tubular epithelial cells, is the most common type of RCC, accounting for $80-90 \%$ of all cases (1). The effect of surgical treatment in patients with circumscribed RCC is good and the 5 -year survival rate is $60-80 \%$ (2). However, the prognosis of patients with distal metastasis remains poor and the median survival time of such patients is only 10 months (2). At the time of diagnosis, 20-35\% of patients already exhibit distal metastasis (3). Furthermore, following surgery, 20-40\% of patients exhibit metastasis, which greatly reduces survival rates. Therefore, metastasis is the main reason for the poor prognosis of patients with RCC and RCC-associated mortality. It has also been hypothesized that RCC staging and pathology are significant factors affecting the prognosis of patients with RCC (4).

The metastasis of malignant cells is dominated by a series of complex physiological procedures. It has been suggested that cell migration serves a key function throughout the process of tumor metastasis, although the mechanism by which this occurs remain unknown (5). In normal epithelial tissue and benign epithelial tumors, epithelial cells are tightly associated with adjacent cells and the basement membrane via tight and adhering junctions, as well as hemidesmosome and desmosome connections (6). In malignant epithelial tumors however, tumor cells are stimulated by external signals and consequently acquire migratory and invasive capabilities. Tumor cells may then degrade the basement membrane, invade the surrounding extracellular matrix and penetrate microvascular or lymphatic vessels. The epithelial-mesenchymal transition (EMT) serves a key function in carcinoma progression, invasion and metastasis. During the EMT, epithelial functions are lost and the expression and distribution of proteins, including E-cadherin, which mediates cell-cell and cell-matrix contacts and the cytoskeletal organization responsible for normal epithelial polarity, become disordered (7). The concurrent gain of mesenchymal characteristics includes the ability of cells to migrate and invade the surrounding matrix due to the increased expression of proteins, including N-cadherin and vimentin (8). However, few studies have investigated the EMT mechanisms that occur in RCC $(5,9)$. Therefore, determining the regulatory mechanisms of the EMT in RCC should improve understanding of how metastasis occurs.

F-box and WD repeat domain containing 7 (Fbxw7) is an F-box protein that belongs to the SKP1-CUL1-F-box 
protein E3 ligase complex and is responsible for transferring the ubiquitin molecule to the substrate, resulting in its recognition and subsequent degradation by proteasomes (10). It identifies a network of signal proteins that function in cell growth, differentiation and apoptosis $(11,12)$. Several specific substrates of Fbxw7 have been identified, including cyclin E (13), c-Jun (14), c-Myc (15), mechanistic target of rapamycin (16) and Notch (17), which are all overexpressed in malignant tumors. Therefore, Fbxw7 is an important tumor suppressor gene, as it negatively regulates the expression of these proteins. The mutation rate of Fbxw7 in cholangiocarcinoma is $35 \%(18,19)$. Furthermore, $30 \%$ of patients with acute $\mathrm{T}$ cell lymphatic leukemia (11), $16 \%$ of patients with primary endometrial cancer (20) and $11 \%$ of patients with colorectal cancer (21) exhibit an Fbxw7 mutation, and the overall mutation frequency of Fbxw 7 is $\sim 6 \%$ in human primary tumors (19). In addition, previous studies have demonstrated that Fbxw7 may affect the migration and invasion of tumors, including gastric cancer and melanoma, and serve as a prognostic marker $(22,23)$. However, to the best of our knowledge, there have been no reports on the association between Fbxw7 and metastasis in RCC.

The aim of the present study was to identify the function of Fbxw7 in the migration and invasion of renal cancer and to investigate its underlying molecular mechanisms of action. Immunohistochemistry (IHC) was used to examine the expression of the Fbxw7 protein in RCC tissues compared with non-cancerous tissue. Subsequently, the potential mechanism of Fbxw7 and the involvement of the EMT in the migration and invasion of RCC cells was explored in vitro.

\section{Materials and methods}

Tissue samples. A total of 70 renal cancer samples and paired normal tumor-adjacent samples were taken from patients (42 males and 28 females, mean age 52.5 years) with RCC who underwent radical nephrectomy or nephron-sparing surgery at the Department of Urology, Ruijin Hospital, Shanghai Jiao Tong University (Shanghai, China) between July 2008 and April 2014. All tumors were pathologically diagnosed as RCC by experienced pathologists (Ruijin Hospital, Shanghai Jiao Tong University School of Medicine, Shanghai, China) by applying the TNM classification (7th edition) (24) following surgery and no patients had received chemo- or radiotherapy prior to surgery. All clinicopathological parameters were acquired from the archive of the pathology department and confirmed by the medical records of the hospital. The present study was approved by the Ethics Committee of the Ruijin Hospital, Shanghai Jiaotong University (Shanghai, China) and all patients gave informed consent for the use of their tissue in the current study. The follow-up period began on the date of tumor resection and ended in February 2014 or once the patient succumbed.

Cell culture. The human RCC cell lines 786-O and ACHN were obtained from the Cell Bank of Type Culture Collection of the Chinese Academy of Sciences (Shanghai, China) and grown in the laboratory. Cells were cultured in RPMI-1640 medium supplemented with $10 \%$ fetal bovine serum (FBS), $100 \mathrm{U} / \mathrm{ml}$ penicillin and streptomycin (all Gibco; Thermo Fisher Scientific
Inc., Waltham, MA, USA), and incubated at $37^{\circ} \mathrm{C}$ in a humidified atmosphere with $5 \% \mathrm{CO}_{2}$.

Western blot analysis. Standard methods were used for western blotting. Cells were lysed in radioimmunoprecipitation assay buffer (Cell Signaling Technology, Inc., Danvers, MA, USA). Protein concentration was determined using a bicinchoninic acid assay kit (Pierce; Thermo Fisher Scientific, Inc.). The total extracted protein contents $(50 \mu \mathrm{g})$ were separated via SDS-PAGE (10\% gel) and then blotted onto a PVDF membrane (EMD Millipore, Billerica, MA, USA). The membrane was blocked with $5 \%$ non-fat milk in PBS with Tween-20 at room temperature for $2 \mathrm{~h}$ and followed by incubation at $4{ }^{\circ} \mathrm{C}$ overnight with primary antibodies against Fbxw7 (cat. no. ab109617; 1:300 dilution; Abcam, Cambridge, MA, USA), E-cadherin (cat. no. 3195; 1:1,000 dilution; Cell Signaling Technology, Inc.), N-cadherin (cat. no. 13116; 1:1,000 dilution; Cell Signaling Technology, Inc.), vimentin (cat. no. 5741; 1:1,000 dilution; Cell Signaling Technology, Inc.) and $\beta$-actin (cat. no. sc70319; 1:1,000 dilution; Santa Cruz Biotechnology, Inc., Dallas, TX, USA). Subsequently, blots were probed with appropriate secondary antibody (cat. no. LI-COR; 1:8,000 dilution; Lincoln, NE, USA) for $1 \mathrm{~h}$ at room temperature and visualized using the Bio-Rad Gel imaging system (Bio-Rad Laboratories, Inc., Hercules, CA, USA). The results were analyzed using Quantity One software (Version 4.62; GelDoc XR+; Bio-Rad Laboratories, Inc.) as specified by Bio-Rad.

Wound healing assay. Cells were seeded to 90-100\% confluence of monolayer cells overnight prior to serum starvation for $8 \mathrm{~h}$ in 6 -well plates at $37^{\circ} \mathrm{C}$. Following wounding with a sterile pipette tip, cells were rinsed three times with PBS to eliminate floating cells. Images of the wounds were captured at 0,24 and $48 \mathrm{~h}$ post-wound using a phase-contrast microscope. The migration rate was calculated as $(\%)=[\mathrm{W}(24$ or $48 \mathrm{~h})-\mathrm{W}(0 \mathrm{~h})] / \mathrm{W}(0 \mathrm{~h})$. All assays were independently performed in triplicate.

Transwell migration and invasion assays. Transwell assays were performed as previously described (25). Cell invasion assays were performed in Matrigel-coated Transwell inserts containing polycarbonate filters with $6-\mu \mathrm{m}$ pores. Cell suspension ( $2 \times 10^{5}$ cells in $200 \mu \mathrm{l}$ serum-free RPMI-1640 medium) was plated onto the upper chamber. In the lower chamber, RPMI 1640 medium containing $10 \%$ FBS was used as a chemoattractant. Following incubation for $24 \mathrm{~h}$ at $37^{\circ} \mathrm{C}$, non-migrating cells were scraped using a cotton swab and cells on the lower surface of the filter were fixed with $4 \%$ methanol for $15 \mathrm{~min}$ at room temperature and stained with $0.5 \%$ crystal violet for $10 \mathrm{~min}$ at room temperature. Cells were counted under a light microscope (magnification, x100) in five different fields and in duplicate wells, in at least three independent experiments. The protocol used for the migration assay was the same as the Matrigel invasion assay, except that the Transwell insert was not coated with Matrigel.

IHC. A total of 70 samples were taken from patients with RCC for which complete clinicopathological information was available. Formalin-fixed, paraffin-embedded tissue sections of $4 \mu \mathrm{m}$ thickness were baked at $60^{\circ} \mathrm{C}$ for $\geq 3 \mathrm{~h}$ and were then 

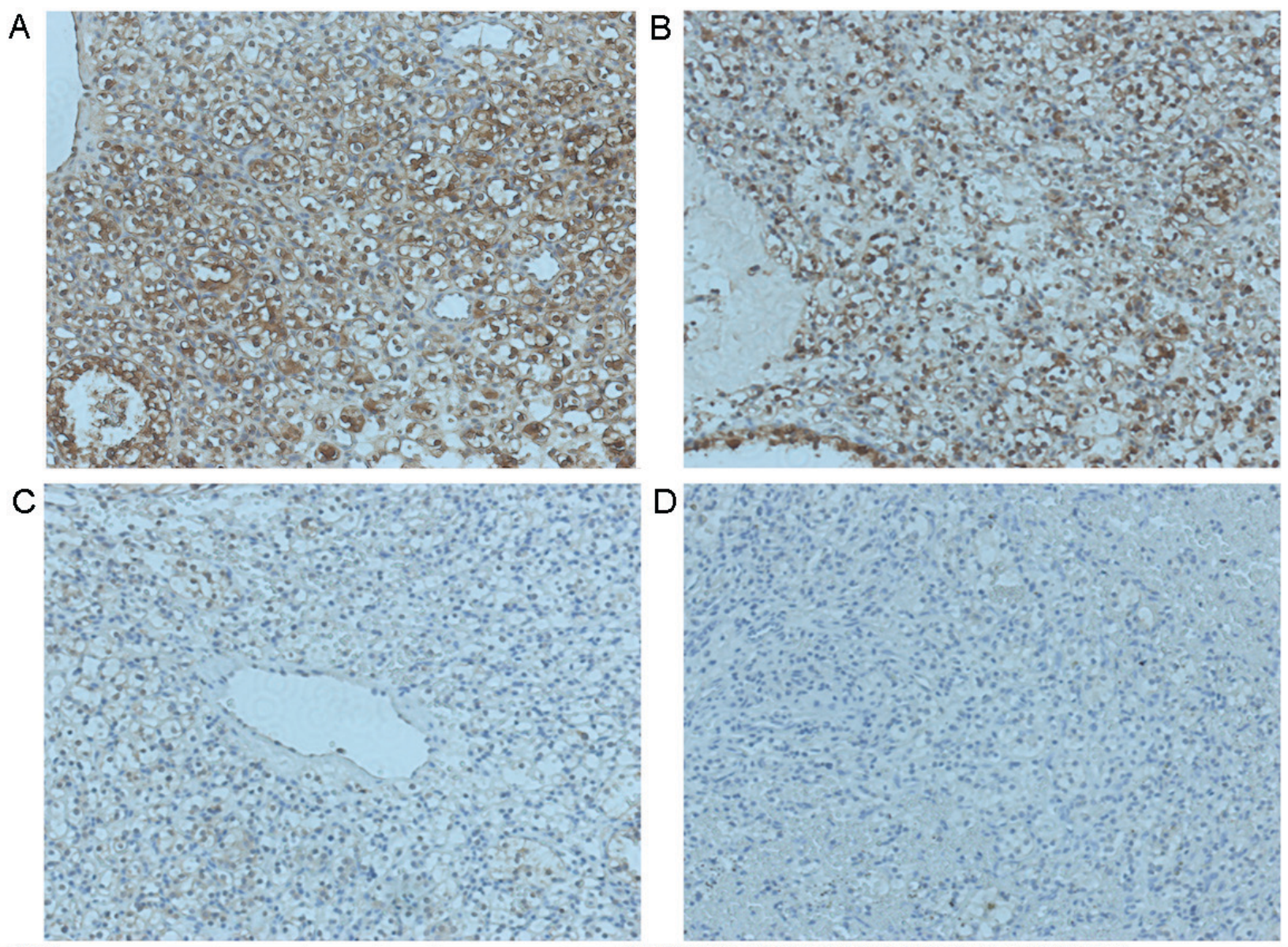

Figure 1. Fbxw7 expression and its clinical significance in patients with RCC. Representative images of Fbxw7 immunohistochemical staining in RCC tissues and corresponding tumor-adjacent tissues. Fbxw7 is (A) markedly positive in tumor-adjacent tissues and (B-D) downregulated in RCC tissues. Positive expression is visualized by brown staining (original magnification, x200). Fbxw7, F-box and WD repeat domain containing 7; RCC, renal cell carcinoma.

incubated twice in xylene for 10 min each time prior to rehydration in a descending alcohol series. Subsequently, citrate buffer was used for antigen retrieval by microwave heating for $15 \mathrm{~min}$. The sections were then blocked in $10 \%$ goat serum (Sp kit; Beijing Solarbio Science \& Technology Co., Ltd., Beijing, China) at $37^{\circ} \mathrm{C}$ for $30 \mathrm{~min}$ prior to incubation at $4^{\circ} \mathrm{C}$ overnight in anti-Fbxw7 primary antibody (cat. no. ab109617; 1:300, Abcam, Cambridge, MA, USA). The biotinylated secondary antibody (Sp kit, Beijing, China) was used to detect the primary antibody at $30^{\circ} \mathrm{C}$ for $30 \mathrm{~min}$. Horseradish peroxidase-streptavidin conjugate was used to connect with biotinylated secondary antibody at $37^{\circ} \mathrm{C}$ for $30 \mathrm{~min}$. The rest of the staining was performed following the manufacturer's protocol. For detection of bound antibody, 3,3'-diaminobenzidine was used for 2-5 min depending on the degree of the color at room temperature. The measurement of positive staining density was determined using an immunohistochemical scoring system to assess the proportion of tumor cells exhibiting specific staining. Staining intensity was assessed using four grades: 0 for none; 1 for weak; 2 for moderate and 3 for strong. The percentage of positive cells was divided into five degrees: 1 for $<5 \%$; 2 for $5-25 \%$; 3 for $26-50 \%$; 4 for $50-75 \%$ and 5 for $75-100 \%$. Total scores $\leq 4$ were defined as low expression and scores of $>4$ were considered to indicate high expression of Fbxw7 (26). Staining was evaluated under a light microscope. The overall intensity and percentage were evaluated by Image-Pro plus software (version 6.0; Media Cybernetics, Rockville, MD, USA) at five independent fields (magnification, x200).

Establishment of knockdown cells and stable expression of Fbxw7. Small interfering RNA (siRNA) specific to human Fbxw7 and scramble siRNA (NC) were purchased from Biomics Biotechnologies Co., Ltd. (Nantong, China). 786-O cells $\left(1 \times 10^{5}\right.$ cells) were plated into 6 -well plates and allowed to adhere for $24 \mathrm{~h}$ and cultured to $60-70 \%$ confluence and cells were then transfected with $100 \mathrm{nM}$ siRNA (Fbxw7 siRNA or NC siRNA) using Lipofectamine 2000 (Invitrogen; Thermo Fisher Scientific, Inc.) according to the manufacturer's protocol. Cells were collected for further investigation $24 \mathrm{~h}$ after transfection.

The plasmid expressing Fbxw7 and the control plasmid (Biomics Biotechnologies, Nantong, China) were transfected into ACHN cells by Lipofectamine 2000 (Invitrogen; Thermo Fisher Scientific, Inc.) when cells were cultured to $80 \%$ confluence according to the manufacturer's protocol. Cells transfected with empty vector (control plasmid) were used as controls. Plasmid-Lipofectamine 2000 complex was prepared as follows: i) Diluted DNA (0.8 g) with $50 \mathrm{ul}$ serum-free medium and mixed gently; ii) diluted $2 \mu \mathrm{l}$ Lipofectamine 2000 with $48 \mu \mathrm{l}$ serum-free medium, mixed gently and incubate for 
Table I. Association of Fbxw7 expression with clinicopathological factors in 70 patients with renal cell carcinoma.

Fbxw7 expression

Patient

clinicopathological parameters

Number of cases

Low expression

High expression

P-values

Age (years)

$\leq 60$

33

$>60$

Sex

Male

Female

28

Histological grade

Well

45

Moderate and poor

Tumor size

$\leq 5 \mathrm{~cm}$

$>5 \mathrm{~cm}$

Tumor stage

I and II

III and IV

Lymph node metastasis

Positive

Negative

Distant metastasis

Positive

Negative
12

58

8

9

12

5

9

8

11

6

5

12

10

7

7

10
0.262

0.856

$0.005^{\mathrm{a}}$

$0.003^{\mathrm{a}}$

25

28

30

23

36

17

33

20

36

17

11

42

$0.006^{\mathrm{a}}$

${ }^{\mathrm{a}} \mathrm{P}<0.05$. Fbxw7, F-box and WD repeat domain containing 7 .

5 min at $4^{\circ} \mathrm{C}$; iii) the two solutions were mixed together and incubated for $20 \mathrm{~min}$ at $4^{\circ} \mathrm{C}$. A total of $100 \mathrm{ul}$ complex was added to the culture plate. The transfected cells were selected and incubated with Geneticin (G418) from Invitrogen (Thermo Fisher Scientific, Inc.) at a dose of $600 \mathrm{ug} / \mathrm{ml}$ for 2 weeks in order to produce overexpression clones. Stable transfected clones were selected and Fbxw7 expression assessed using western blot analysis.

Statistical analysis. All data are presented as the mean \pm standard deviation from $\geq 3$ separate experiments. A $\chi^{2}$ test was used to analyze the association between Fbxw7 expression and patient clinicopathological characteristics. The Kaplan-Meier survival curve was used to analyze overall survival (OS) rates of patients and differences were compared using the Log-rank test. Cox proportional hazard analysis was employed for univariate and multivariate analyses. ${ }^{*} \mathrm{P}<0.05$ was considered to indicate a statistically significant difference. Numerical data were calculated using Microsoft Excel and analyzed using SPSS 18.0 (SPSS, Inc., Chicago, IL, USA).

\section{Results}

Fbxw7 is frequently downregulated in RCC and is associated with poor RCC prognosis. IHC was performed to
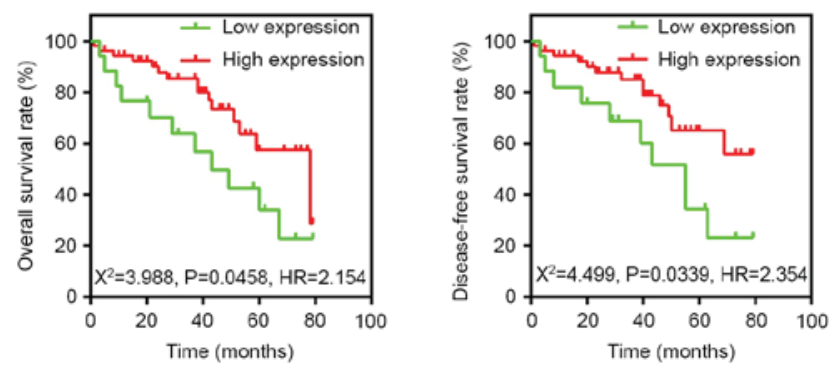

Figure 2. Kaplan-Meier 5-year overall and disease-free survival curves in 70 patients with RCC according to Fbxw7 protein expression. Patients with RCC and low Fbxw7 expression exhibit poorer prognosis following surgery compared with those expressing higher levels of Fbxw7 $(\mathrm{P}<0.05$, log-rank test). Fbxw7, F-box and WD repeat domain containing 7; RCC, renal cell carcinoma.

investigate the expression of Fbxw7 in tumor and adjacent non-tumor tissues collected from 70 patients with RCC to determine the clinical significance of Fbxw7 (Fig. 1). Of the 70 tissues analyzed, Fbxw7 expression was downregulated in 17 RCC samples (24.3\%) compared with adjacent non-tumor tissues. Additionally, to determine the association between Fbxw7 expression and the clinicopathological features of patients with RCC, 70 patients with RCC were separated 
Table II. Univariate and multivariate Cox regression analyses of overall survival in 70 patients with renal cell carcinoma.

\begin{tabular}{llr}
\hline Tumor characteristics & HR $(95 \%$ CI) & P-value \\
\hline Univariate analysis & & \\
Age ( $\geq 60$ vs. $<60)$ years & $1.461(0.943-2.265)$ & 0.090 \\
Sex (male vs. female) & $0.978(0.575-1.664)$ & 0.935 \\
Histological grade (poor/well) & $1.784(1.130-2.818)$ & $0.013^{\mathrm{a}}$ \\
Tumor size (>5 vs. $\leq 5) \mathrm{cm}$ & $1.454(0.950-2.227)$ & 0.085 \\
Tumor stage (III, IV/I, II) & $2.512(2.112-2.987)$ & $<0.001^{\mathrm{a}}$ \\
Lymph node metastasis (positive vs. negative) & $2.336(1.966-2.776)$ & $<0.001^{\mathrm{a}}$ \\
Distant metastasis (positive vs. negative) & $2.621(1.984-3.462)$ & $<0.001^{\mathrm{a}}$ \\
Fbxw7 (high vs. low) & $0.465(0.360-0.601)$ & $<0.001^{\mathrm{a}}$ \\
Multivariate analysis & & $<.124$ \\
Histological grade (poor /well) & $1.436(0.906-2.275)$ & $<0.001^{\mathrm{a}}$ \\
Tumor stage (III, IV/I, II) & $1.962(1.626-2.369)$ & $<0.001^{\mathrm{a}}$ \\
Lymph node metastasis (positive vs. negative) & $1.784(1.459-2.181)$ & $<0.001^{\mathrm{a}}$ \\
Distant metastasis (positive vs. negative) & $1.621(1.225-2.145)$ & $<0.001^{\mathrm{a}}$ \\
Fbxw7 (high vs. low) & $0.623(0.479-0.810)$ &
\end{tabular}

${ }^{\mathrm{a}} \mathrm{P}<0.05$. HRs, hazard ratios; CI, confidence interval.

into Fbxw7 low and high expression groups, according to their IHC scores. The results revealed a significant association between Fbxw7 expression and tumor node metastasis (TNM) (24) stage $(\mathrm{P}=0.005)$, lymph node metastasis $(\mathrm{P}=0.003)$ and distant metastasis $(\mathrm{P}=0.006)$. However, there were no significant associations between Fbxw7 expression and other clinicopathological factors, including age, gender, tumor size and histological grade (Table I). These data indicate that the decreased expression of Fbxw7 stimulates RCC progression.

Low Fbxw7 expression is associated with the poor survival of patients with RCC. To determine whether the decreased expression of Fbxw7 in RCC was associated with poor patient prognosis, a Kaplan-Meier survival analysis was performed using the overall 5-year survival and disease-free survival data from 70 patients with RCC (Fig. 2). The results revealed that patients with low Fbxw7 expression had a significantly decreased postoperative survival time compared with those with high Fbxw7 expression ( $\mathrm{P}=0.0458)$. Furthermore, Fbxw7 downregulation was associated with a significantly shorter disease-free survival duration $(\mathrm{P}=0.0339)$. To control for potential confounders, a Cox proportional hazards regression analysis was performed, which revealed that histological grade $(\mathrm{P}=0.013)$, Fbxw7 expression $(\mathrm{P}<0.001)$, tumor stage $(\mathrm{P}<0.001)$, lymph node metastasis $(\mathrm{P}<0.001)$ and distant metastasis $(\mathrm{P}<0.001)$ were significantly associated with the OS of patients with RCC (Table II). Multivariate analysis further confirmed that reduced $\mathrm{Fbxw} 7$ expression was an independent predictor for a shorter OS in patients with RCC $(\mathrm{HR}=0.623 ; \mathrm{P}<0.001$; Table II). These data indicated that reduced Fbxw7 expression is associated with the poorer prognosis of patients with RCC.

Fbxw7 regulates the migration and invasion of $R C C$ cells. The ability to migrate and invade is important for RCC

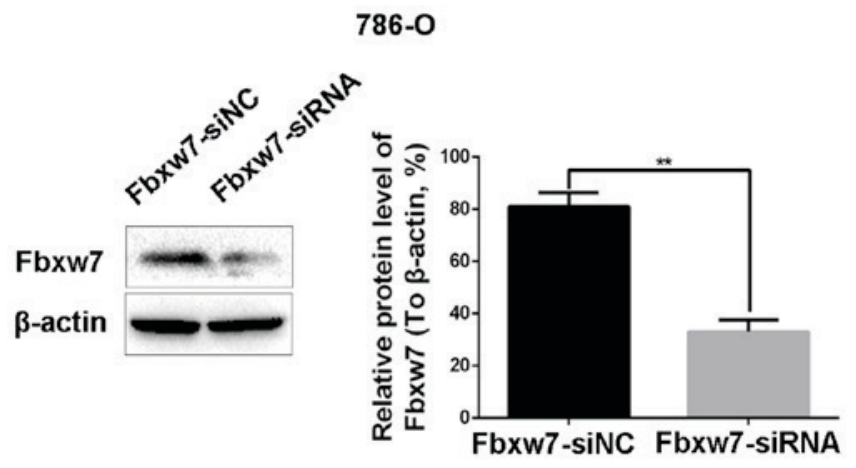

Figure 3. Downregulation of Fbxw7 increases the migration and invasion of 786-O cells in vitro. The effect of Fbxw7 siRNA interference on Fbxw7 expression in $786-\mathrm{O}$ cells was confirmed by western blot analysis. ${ }^{* *} \mathrm{P}<0.01$ with comparisons shown by lines. Fbxw7, F-box and WD repeat domain containing 7; NC, negative control; siRNA, small interfering RNA.

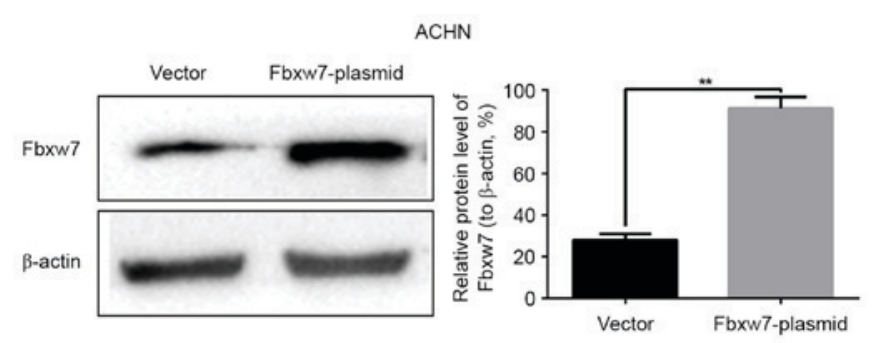

Figure 4. Upregulation of Fbxw7 decreases the migration and invasion of ACHN cells in vitro. The effect of Fbxw7 plasmid transfection on Fbxw7 expression in ACHN cells was confirmed by western blot analysis. ${ }^{* *} \mathrm{P}<0.01$ with comparisons shown by lines. Fbxw7, F-box and WD repeat domain containing 7 .

metastasis. Currently, little is known regarding whether Fbxw7 is able to regulate the migration and invasion of 


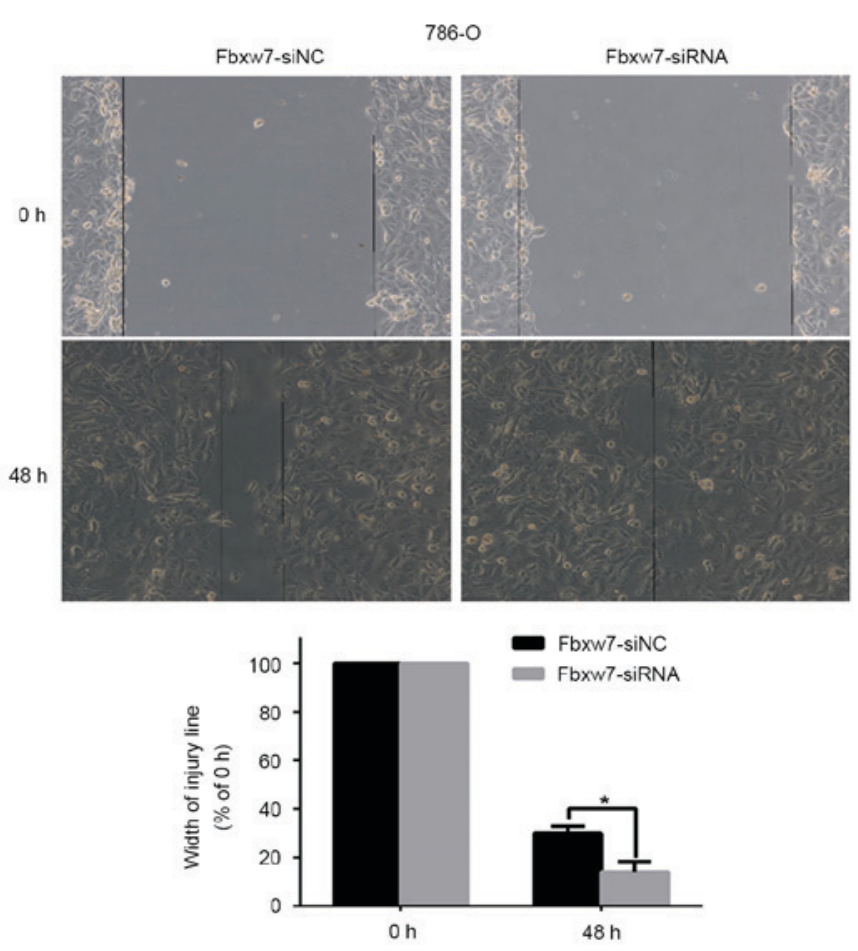

Figure 5. Migration of Fbxw7-silencing 786-O cells and its control cells were measured using a wound-healing assay (Magnification, $\mathrm{x} 100$ ). ${ }^{*} \mathrm{P}<0.05$ with comparisons shown by lines. Fbxw7, F-box and WD repeat domain containing 7; NC, negative control; siRNA, small interfering RNA.

RCC cells. Thus, to identify whether Fbxw7 regulates these processes in RCC, the 786-O and ACHN cell lines were employed, since it has been demonstrated that the expression of Fbxw7 is high in 786-O cells and low in ACHN cells (26). The expression of Fbxw7 in the 786-O cell line was downregulated using Fbxw7-siRNA and upregulated in the ACHN cell line using an Fbxw7 expressing plasmid. Their respective negative controls were also established. Changes in Fbxw7 expression in the two cell lines following transfection were confirmed by western blotting (Figs. 3 and 4). Subsequently, wound healing and Transwell migration assays were used to assess the migration ability of transfected cells and the Transwell invasion assay was employed to assess the invasive ability of the cells.

To determine whether Fbxw7 affects RCC cell migration, a wound healing assay was performed, which revealed that Fbxw7 silencing significantly reduced the wound healing time in 786-O cells compared with negative controls at $48 \mathrm{~h}$ ( $\mathrm{P}=0.047$; Fig. 5). By contrast, the wound healing time in ACHN cells significantly increased following Fbxw7 overexpression compared with cells in the control group at $48 \mathrm{~h}$ $(\mathrm{P}=0.027$; Fig. 6). Furthermore, a Transwell assay without Matrigel was performed, which revealed that the number of Fbxw7-siRNA-transfected 786-O cells migrating through the membrane partition into the lower chamber was significantly higher than the vector group ( $\mathrm{P}=0.0027$; Fig. 7). By contrast, Fbxw7-overexpressing ACHN cells exhibited a significantly lower migration rate compared with the control group ( $\mathrm{P}=0.037$; Fig. 8).

A Transwell assay with Matrigel was also performed to elucidate the function of Fbxw7 in tumor cell invasion. There

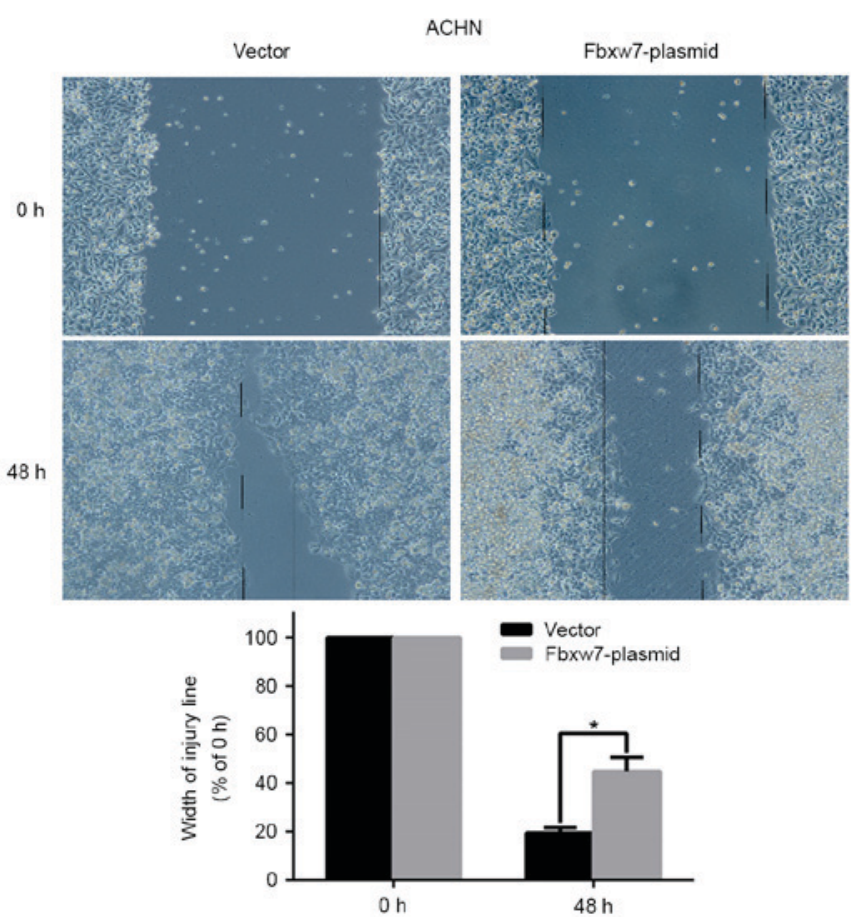

Figure 6. Migration of Fbxw7-overexpressing ACHN cells and its control cells were measured by the wound-healing assay (Magnification, $\mathrm{x} 100$ ). ${ }^{*} \mathrm{P}<0.05$ with comparisons shown by lines. Fbxw7, F-box and WD repeat domain containing 7 .

were significantly more $786-\mathrm{O}$ cells invading through the Matrigel in the Fbxw7-siRNA group than in the Fbxw7-NC siRNA group ( $\mathrm{P}=0.0028$; Fig. 7). However, elevated Fbxw7 expression significantly reduced the number of ACHN cells that invaded through the Matrigel compared with the control cells ( $\mathrm{P}=0.029$; Fig. 8). These results indicate that Fbxw7 inhibits the migration and invasion of RCC cells.

Fbxw 7 regulates the EMT in RCC cells. From the aforementioned results, it was concluded that the decreased expression of Fbxw7 stimulates the metastasis of RCC cells. Therefore, it was investigated whether Fbxw7 influences the invasive behavior of RCC cells via regulation of the EMT. Western blotting identified a significant decrease in the expression of the epithelial marker protein E-cadherin in 786-O cells following Fbxw7 silencing. It also identified a significant increase in the expression of the mesenchymal marker proteins $\mathrm{N}$-cadherin and vimentin $(\mathrm{P}<0.05$; Fig. 9). By contrast, the overexpression of Fbxw7 in ACHN cells significantly increased E-cadherin expression and significantly reduced the expression of $\mathrm{N}$-cadherin and vimentin $(\mathrm{P}<0.05$; Fig. 10). This suggests that Fbxw7 inhibits the EMT. Overall, these results suggest that the downregulation of Fbxw7 in RCC accelerates cell migration and invasion by inducing the EMT.

\section{Discussion}

RCC is a common human malignancy, accounting for $2-3 \%$ of adult malignant tumors. Treatment is effective when RCC is limited to the kidney and the postoperative 5-year survival 
786-O
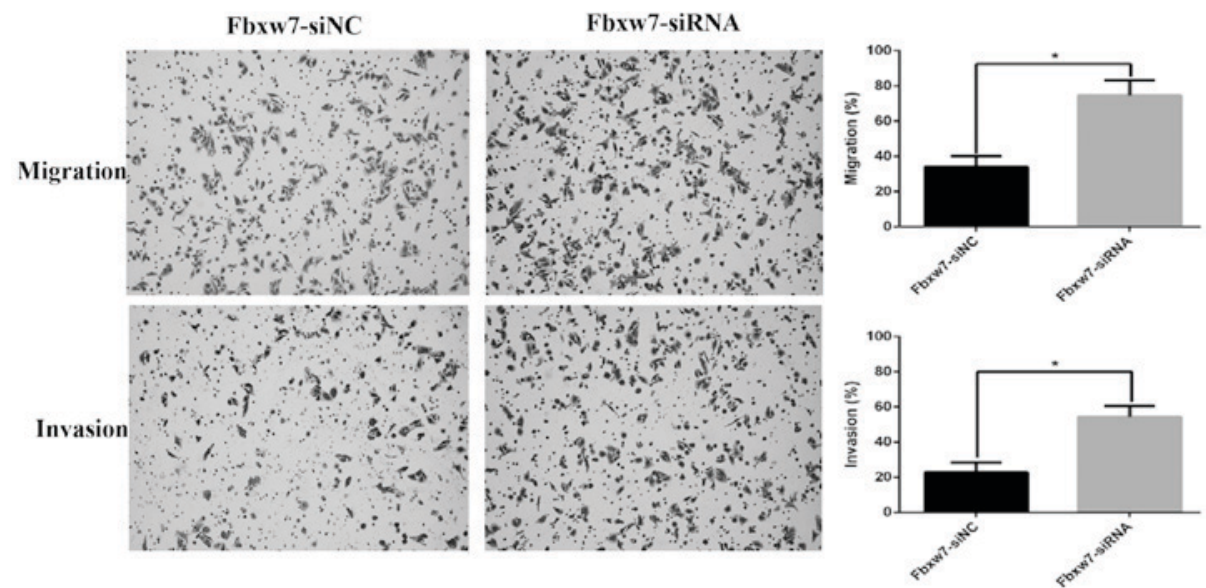

Figure 7. The migratory and invasive ability of Fbxw7-silenced 786-O cells and control cells were determined using the uncoated or Matrigel-coated Transwell assay " $\mathrm{P}<0.05$ with comparisons shown by lines. (Magnification, $\mathrm{x} 200$ ). Fbxw7, F-box and WD repeat domain containing 7; NC, negative control; siRNA, small interfering RNA.
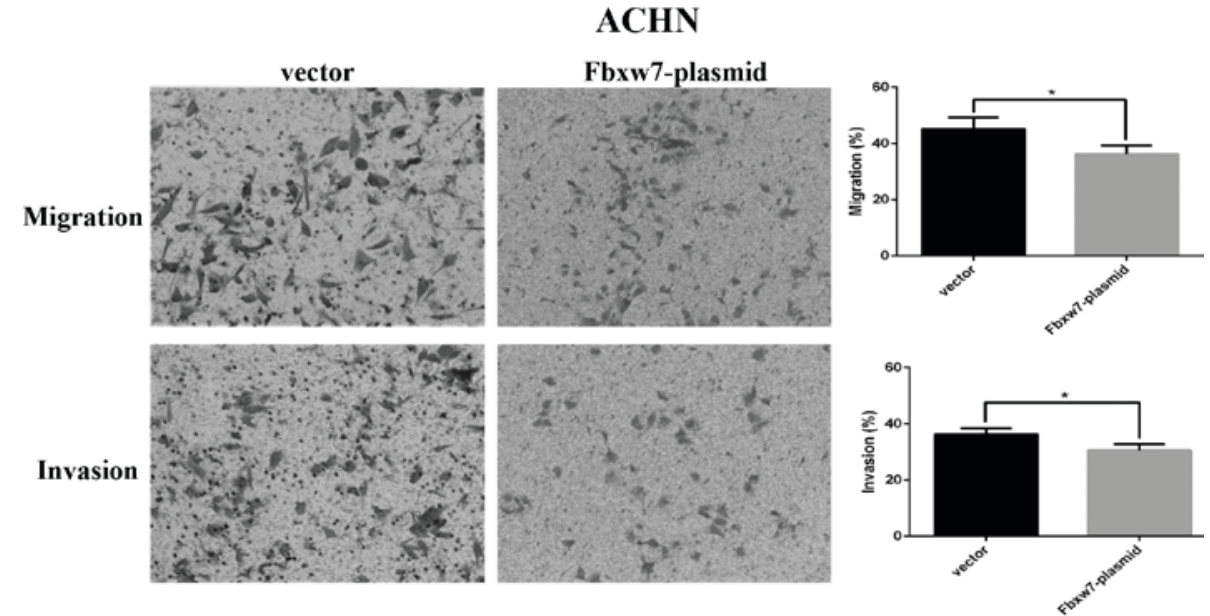

Figure 8. Migration and invasion ability of Fbxw7-overexpressing ACHN cells and its control cells were determined using an uncoated or Matrigel-coated Transwell assay " $\mathrm{P}<0.05$ with comparisons shown by lines. Fbxw7, F-box and WD repeat domain containing 7.

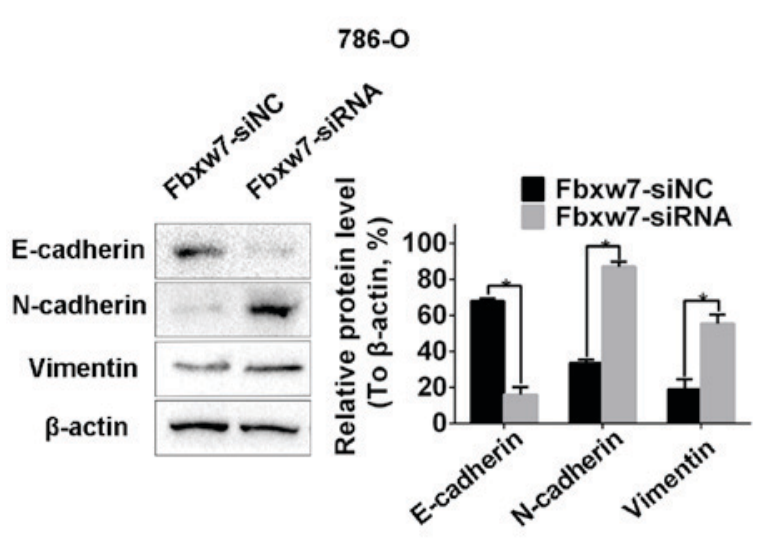

Figure 9. Fbxw7 serves crucial roles in the epithelial-mesenchymal transition in renal cell carcinoma. Western blot analysis demonstrating increased expression of $\mathrm{N}$-cadherin, vimentin and decreased expression of E-cadherin following Fbxw7 downregulation in 786-O cells. ${ }^{*} \mathrm{P}<0.05$ with comparisons shown by lines. Fbxw7, F-box and WD repeat domain containing 7; NC, negative control; siRNA, small interfering RNA. rate is $60-80 \%$ in such cases. However, following the onset of metastasis, the prognosis of patients worsens and such patients exhibit a median survival time of $\sim 10$ months. Therefore, metastasis is an important cause of poor prognosis (4). However, the mechanisms underlying the progression and metastasis of RCC remain unclear.

Previous studies have demonstrated that Fbxw7 serves a key role as a suppressor gene in the cell cycle, proliferation, differentiation, apoptosis, tumor metastasis and drug resistance $(11,12)$. Clinical and basic studies have demonstrated that the decreased expression or mutations of Fbxw7 are common in all types of human tumors, including $\mathrm{T}$ cell acute lymphoblastic leukemia, pancreatic, gastric, colorectal and prostate cancer, cholangiocarcinoma and endometrial cancer $(11,19-21)$, with an overall mutation rate of $\sim 6 \%(19,27)$. In addition, there are a number of studies investigating the role of Fbxw7 in tumor cell migration and metastasis, including in gastric cancer, hepatocellular carcinoma and hepatocholangiocarcinoma $(22,23)$. It has been 


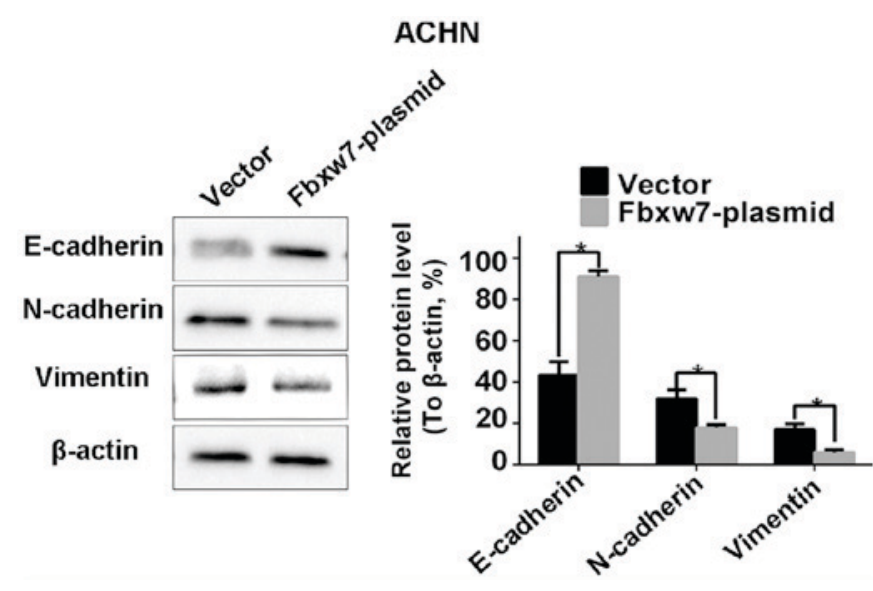

Figure 10. Fbxw7-overexpressing ACHN cells exhibited decreased expression of $\mathrm{N}$-cadherin, vimentin and increased expression of E-cadherin. ${ }^{*} \mathrm{P}<0.05$ with comparisons shown by lines. Fbxw7, F-box and WD repeat domain containing 7 .

demonstrated that the level of Fbxw7 is decreased in renal clear cancer cells and that the ectopic expression of Fbxw7 may inhibit the proliferation and induce the apoptosis of tumor cells (26). In the present study, the role of Fbxw7 in RCC cell migration and invasion was investigated. Initially, the expression of Fbxw7 in RCC tissues was detected by IHC and in combination with clinical pathological parameters, the results indicated that low Fbxw7 expression in tumor tissues was significantly associated with tumor stage, lymph node and distant metastases. In addition, clinical prognostic analysis revealed a significantly improved OS rate in patients with high expression of Fbxw7. Additionally, multivariate Cox regression analyses demonstrated that the low expression of Fbxw7 was associated with poor patient prognosis and may be an independent predictor of poor prognosis for patients with RCC. Due to the limited variables in the current study, multivariate analysis cannot control all the potential confounders. Therefore, potential limitations existed in the results that require verification in further studies. Although these observations highlight the role of Fbxw7 in the progression of RCC, to the best of our knowledge, the current study is the first to investigate this function of Fbxw7 and its precise mechanisms in the regulation of migration and invasion in RCC cells. Therefore, the results of the current study indicate that Gbxw7 may be used to diagnose and treat patients with RCC.

In order to verify the influence of Fbxw7 on the ability of RCC cells to invade and migrate, Fbxw7 knockdown and overexpression were performed in 786-O and ACHN cells, respectively. The migration and invasion of tumor cells was evaluated using Transwell and wound healing assays. The Transwell assay is a common method to detect the ability of cell invasion and migration in vitro. The principle is that cells in the upper chamber are cultured in a serum-free medium and culture medium containing 10\% FBS is added to the lower chamber (28). There are two main reasons why low nutrient culture medium is added to the upper chamber: Cells in the relatively low nutrient culture exhibit a reduction in proliferation; by contrast, tumor cells are spontaneously move to the lower chamber containing higher FBS, which acts as a chemoattractant. Under the same conditions, more tumor cells with a higher migration ability may migrate to the lower chamber than tumor cells and with a weaker ability to migrate. Adding Matrigel to the upper chamber means that tumor cells must degrade the Matrigel through the chamber to simulate the metastatic process to allow tumor cells to invade through the basement membrane. Therefore, it can evaluate the invasive ability of cells. In the present study, the results of the wound healing and Transwell migratory assays revealed that the migratory ability of 786-O cells following Fbxw7 knockdown increased significantly. Furthermore, the results of the Transwell invasion assay demonstrated that the invasive ability of 786-O cells increased significantly following Fbxw7 knockdown. By contrast, the invasion and migration ability of ACHN cells with high levels of Fbxw7 was evidently weakened. These results indicate that Fbxw7 inhibits RCC cell invasion and metastasis. To the best of our knowledge, the present study is the first to report that Fbxw7 downregulation promotes the invasion and metastasis of RCC cells.

It has been suggested that tumor metastasis is a multi-step biochemical process with numerous molecular events (29). Firstly, adhesion of tumor cells decreases, resulting in tumor cells separating from each other. Subsequently, tumor cells adhere to the extracellular matrix by binding to receptors on the cell surface; eventually, tumor cells may migrate and invade to the surrounding tissues by degrading the extracellular matrix $(30,31)$. The proliferation, adhesion and migration of tumor cells serve an important role during the different stages of invasion. The EMT is one of the most important mechanisms of tumor invasion and metastasis in which the adhesion protein E-cadherin is critical. In the present study, enhanced Fbxw7 expression significantly attenuated the migration and invasion of RCC cells. Additionally, when Fbxw7 expression was repressed using a specific siRNA, the migration and invasion of tumor cells was significantly increased. Furthermore, the association between Fbxw7 and the EMT process was also explored. The results revealed that the EMT phenotypes were reversed in the RCC cells following overexpression of Fbxw7 in ACHN cells or knockdown of Fbxw7 in 786-O cells, as indicated by the altered expression of epithelial and mesenchymal biomarkers. These results reveal that Fbxw7 regulates RCC cell metastasis by modulating the EMT. Consistent with the data analysis of the clinical characteristics in the present study, these results support the hypothesis that Fbxw7 serves a significant role in inhibiting the migration and invasion of RCC cells.

In conclusion, the present study demonstrated that the decreased expression of Fbxw7 is associated with RCC progression and poor patient prognosis and indicated that its underlying mechanism of action is associated with regulating the EMT. Therefore, based on the results of in vitro experiments investigating the migration and invasion of RCC cells, the results of the present study indicate that Fbxw7 may be used as a potential target against tumor metastasis in RCC. Furthermore Fbxw7 may be used as an additional indicator to improve the prognosis of patients with RCC. However, the function of Fbxw7 in RCC remains unclear and further studies are required. 


\section{References}

1. Siegel R, DeSantis C, Virgo K, Stein K, Mariotto A, Smith T, Cooper D, Gansler T, Lerr C, Fedewa S, et al: Cancer treatment and survivorship statistics, 2012. CA Cancer J Clin 62: 220-241, 2012.

2. Sun BL, Chen L, Fu H, Guo L, Guo H and Zhang N: Upregulation of RICTOR gene transcription by the proinflammatory cytokines through NF- $x \mathrm{~B}$ pathway contributes to the metastasis of renal cell carcinoma. Tumour Biol 37: 4457-4466, 2016

3. Finley DS, Pantuck AJ and Belldegrun AS: Tumor biology and prognostic factors in renal cell carcinoma. Oncologist 16 (Suppl 2): S4-S13, 2011.

4. Xiong J, Liu Y, Jiang L, Zeng Y and Tang W: High expression of long non-coding RNA lncRNA-ATB is correlated with metastases and promotes cell migration and invasion in renal cell carcinoma. Jpn J Clin Oncol 46: 378-384, 2016.

5. Song J, Chen X, Bai J, Liu Q, Li H, Xie J, Jing H and Zheng J: Discoidin domain receptor 1 (DDR1), a promising biomarker, induces epithelial to mesenchymal transition in renal cancer cells. Tumour Biol 37: 11509-11521, 2016.

6. Lauffenburger D and Horwitz AF: Cell migration: A physically integrated molecular process. Cell 84: 359-369, 1996.

7. Lee J: The epithelial-mesenchymal transition: New insights in signaling, development and disease. J Cell Biol, . 172: 973-981, 2006.

8. Thiery JP and Sleeman JP: Complex networks orchestrate epithelial-mesenchymal transitions. Nat Rev Mol Cell Biol 7: 131-142, 2006.

9. Li Q, Hou L, Ding G, Li Y, Wang J, Qian B, Sun J and Wang Q: KDM6B induces epithelial-mesenchymal transition and enhances clear cell renal cell carcinoma metastasis through the activation of SLUG. Int J Clin Exp Pathol 8: 6334-6344, 2015.

10. Wang Z, Inuzuka H, Fukushima H, Wan L, Gao D, Shaik S, Sarkar FH and Wei W: Emerging roles of the FBW7 tumour suppressor in stem cell differentiation. EMBO Res 13: 36-43, 2011.

11. Welcker M and Clurman BE: FBW7 ubiquitin ligase: A tumour suppressor at the crossroads of cell division, growth and differentiation. Nat Rev Cancer 8: 83-93, 2008.

12. Wang Z, Inuzuka H, Zhong J, Wan L, Fukushima H, Sarkar FH and Wei W: Tumor suppressor functions of FBW7 in cancer development and progression. FEBS Lett 586: 1409-1418, 2012.

13. Koepp DM, Schaefer LK, Ye X, Keyomarsi K, Chu C, Harper JW and Elledge SJ: Phosphorylation-dependent ubiquitination of cyclin E by the SCFFbw7 ubiquitin ligase. Science 294: 173-177, 2001.

14. Wei W, Jin J, Schlisio S, Harper JW and Kaelin WG Jr: The v-Jun point mutation allows c-Jun to escape GSK3-dependent recognition and destruction by the Fbw7 ubiquitin ligase. Cancer Cell 8: 25-33, 2005.

15. Welcker M, Orian A, Jin J, Grim JE, Harper JW, Eisenman RN and Clurman BE: The Fbw7 tumor suppressor regulates glycogen synthase kinase 3 phosphorylation-dependent c-Myc protein degradation. Proc Natl Acad Sci USA 101: 9085-9090, 2004.

16. Mao JH, Kim IJ, Wu D, Climent J, Kang HC, DelRosario R and Balmain A: FBXW7 targets mTOR for degradation and cooperates with PTEN in tumor suppression. Science 321: 1499-1502, 2008.

17. Gupta-Rossi N, Le Bail O, Gonen H, Brou C, Logeat F, Six E, Ciechanover A and Israël A: Functional interaction between SEL-10, an F-box protein, and the nuclear form of activated Notch1 receptor. J Biol Chem 276: 34371-34378, 2001.
18. Thompson BJ, Buonamici S, Sulis ML, Palomero T, Vilimas T, Basso G, Ferrando A and Aifantis I: The SCFFBW7 ubiquitin ligase complex as a tumor suppressor in T cell leukemia. J Exp Med 204: 1825-1835, 2007.

19. Akhoondi S, Sun D, von der Lehr N, Apostolidou S, Klotz K, Maljukova A, Cepeda D, Fiegl H, Dafou D, Marth C, et al: FBXW7/hCDC4 is a general tumor suppressor in human cancer. Cancer Res 67: 9006-9012, 2007.

20. Hubalek MM, Widschwendter A, Erdel M, Gschwendtner A, Fiegl HM, Müller HM, Goebel G, Mueller-Holzner E, Marth C, Spruck CH, et al: Cyclin E dysregulation and chromosomal instability in endometrial cancer. Oncogene 23: 4187-4192, 2004.

21. Lee JW, Soung YH, Kim HJ, Park WS, Nam SW, Kim SH, Lee JY, Yoo NJ and Lee SH: Mutational analysis of the hCDC4 gene in gastric carcinomas. Eur J Cancer 42: 2369-2373, 2006.

22. Li J, Guo Y, Liang X, Sun M, Wang G, De W and Wu W: MicroRNA-223 functions as an oncogene in human gastric cancer by targeting $\mathrm{FBXW} 7 / \mathrm{hCdc} 4$. J Cancer Res Clin Oncol 138: 763-774, 2012.

23. Cheng Y, Chen G, Martinka M, Ho V and Li G: Prognostic significance of Fbw7 in human melanoma and its role in cell migration. J Invest Dermatol 133: 1794-1802, 2013.

24. Santiago JM, Sasako M and Osorio J: TNM-7th edition 2009 (UICC/AJCC) and Japanese Classification 2010 in Gastric Cancer. Towards simplicity and standardisation in the management of gastric cancer. Cir Esp 89: 275-281, 2011 (In Spanish).

25. Xu W, Wang Z, Zhang W, Qian K, Li H, Kong D, Li Y and Tang Y: Mutated K-ras activates CDK8 to stimulate the epithelial-to-mesenchymal transition in pancreatic cancer in part via the Wnt/ $\beta$-catenin signaling pathwa. Cancer Lett 356: 613-627, 2015.

26. Fu Y, Lin Y, Yang Z, Yang G, Li G, Liu Y, Tan X, Huang Y, Wu X, Wang Y, et al: FBXW7 overexpression suppresses renal cancer cell proliferation and induces apoptosis. Med Oncol 32: 215,2015

27. Wang Y, Liu Y, Lu J, Zhang P, Wang Y, Xu Y, Wang Z, Mao JH and Wei G: Rapamycin inhibits FBXW7 loss-induced epithelial-mesenchymal transition and cancer stem cell-like characteristics in colorectal cancer cells. Biochem Biophys Res Commun 434: 352-356, 2013.

28. Cao J, Liu J, Xu R, Zhu X, Liu L and Zhao X: MicroRNA-21 stimulates epithelial-to-mesenchymal transition and tumorigenesis in clear cell renal cells. Mol Med Rep 13: 75-82, 2016.

29. Song Y, Washington MK and Crawford HC: Loss of FOXA1/2 is essential for the epithelial-to-mesenchymal transition in pancreatic cancer. Cancer Res 70: 2115-2125, 2010.

30. Yang $\mathbf{J}$ and Weinberg RA: Epithelial-mesenchymal transition: At the crossroads of development and tumor metastasis. Dev Cell 14: 818-829, 2008.

31. Schmalhofer O, Brabletz $\mathrm{S}$ and Brabletz T: E-cadherin, beta-catenin, and ZEB1 in malignant progression of cancer. Cancer Metastasis Rev 28: 151-166, 2009.

This work is licensed under a Creative Commons Attribution-NonCommercial-NoDerivatives 4.0 International (CC BY-NC-ND 4.0) License. 